\title{
DISCRETE REFLEXIVITY IN GO SPACES
}

\author{
Vladimir V. TKachuk and Richard G. Wilson \\ Universidad Autónoma Metropolitana, Mexico City, Mexico
}

\begin{abstract}
A property $\mathcal{P}$ is discretely reflexive if a space $X$ has $\mathcal{P}$ whenever $\bar{D}$ has $\mathcal{P}$ for any discrete set $D \subset X$. We prove that quite a few topological properties are discretely reflexive in GO spaces. In particular, if $X$ is a GO space and $\bar{D}$ is first countable (paracompact, Lindelöf, sequential or Fréchet-Urysohn) for any discrete $D \subset X$ then $X$ is first countable (paracompact, Lindelöf, sequential or Fréchet-Urysohn respectively). We show that a space with a nested local base at every point is discretely locally compact if and only if it is locally compact. Therefore local compactness is discretely reflexive in GO spaces. It is shown that a GO space is scattered if and only if it is discretely scattered. Under $\mathrm{CH}$ we show that Cech-completeness is not discretely reflexive even in second countable linearly ordered spaces. However, discrete Cech-completeness of $X \times X$ is equivalent to its Cech-completeness if $X$ is a LOTS. We also establish that any discretely Čech-complete Borel set must be Čechcomplete.
\end{abstract}

\section{INTRODUCTION}

Say that a topological property $\mathcal{P}$ is discretely reflexive in a class $\mathcal{A}$ if a space $X \in \mathcal{A}$ has $\mathcal{P}$ if and only if the closure of every discrete subspace of $X$ has $\mathcal{P}$. Tkachuk proved in [18] that compactness is discretely reflexive in any space and a systematic study of discrete reflexivity was undertaken in [1]. The paper [1] also contains results on discrete reflexivity in compact spaces. Tkachuk and Burke showed in [7] that several properties are discretely reflexive in countably compact spaces.

2010 Mathematics Subject Classification. 54D45, 54F05, 54G12.

Key words and phrases. Discretely reflexive property, discretely Lindelöf space, GO space, discretely locally compact space, discretely Čech-complete space, $d$-separable space, discretely scattered space, linearly ordered space. 
If we consider completeness or convergence properties then one cannot expect many positive results in general spaces. Indeed, van Douwen's example of countable maximal space [10, Example 3.3] shows that a space $X$ need not be sequential or have the Baire property even if every discrete subspace in $X$ is closed and hence $X$ is discretely metrizable and discretely Cech-complete at the same time.

In this paper we deal with discrete reflexivity in spaces with an order-type structure showing that quite a few properties are discretely reflexive in such spaces. For example tightness, character, pseudocharacter, sequentiality and the property of being a $k$-space (which henceforth we will call the $k$-property) are discretely reflexive in GO spaces.

It is an open problem of Arhangel'skii ([3, Problem 14]) whether the Lindelöf property is discretely reflexive. Arhangel'skii and Buzyakova established in [4] that any discretely Lindelöf space of countable tightness is Lindelöf. We show that paracompactness and Lindelöfness are both discretely reflexive in GO spaces.

It turns out that the situation with discrete reflexivity of completeness properties is not trivial even in second countable spaces. We show that, under $\mathrm{CH}$, a dense subset $X$ of the real line $\mathbb{R}$ need not be Cech-complete while $\bar{D}$ is Čech-complete for any discrete $D \subset X$. However, if $X$ is a Borel set then it is Čech-complete if and only if so is $\bar{D}$ for any discrete $D \subset X$. We establish that a space with a nested base at every point is locally compact if and only if it is discretely locally compact. This result is new even for GO spaces. It also turns out that being scattered is a reflexive property both in metrizable spaces and in GO spaces.

Juhasz and Szentmiklossy established in [16] that, for any compact space $X$, there exists a discrete subspace $D \subset X \times X$ such that $|D|=d(X)$. Burke and Tkachuk generalized this result in [6] proving that for any Lindelöf $p$-space $X$ there is a discrete $D \subset X \times X$ such that $\Delta_{X} \subset \bar{D}$. This result has many applications in the study of discrete reflexivity. In this paper we prove that if $X$ is a linearly ordered topological space then there is a discrete $D \subset X \times X$ such that $\Delta_{X} \subset \bar{D}$. As a consequence, $X^{\omega}$ is $d$-separable; besides, if $X \times X$ is discretely Čech-complete (discretely metrizable) then $X$ is Čech-complete (metrizable respectively).

\section{NOTATION AND TERMinOLOGY}

All spaces are assumed to be Tychonoff. If $X$ is a space then $\tau(X)$ is its topology; given any point $x \in X$ let $\tau(x, X)=\{U \in \tau(X): x \in U\}$. The set $\mathbb{R}$ is the real line with its usual topology and $\mathbb{Q} \subset \mathbb{R}$ is the set of rationals. If $X$ is a space then $\Delta_{X}=\{(x, x): x \in X\}$ is its diagonal; we write $\Delta$ instead of $\Delta_{X}$ if $X$ is clear. A space $X$ is called a Borel set if it is homeomorphic to a Borel subset of $\mathbb{R}^{\omega}$. Call $X$ a Lindelöf $p$-space if there exists a perfect map of 
$X$ onto a second countable space. A space $X$ is $d$-separable if it has a dense $\sigma$-discrete subset.

If we are given a linearly ordered set $(X,<)$ and $a, b \in X$ then, as usual, $(a, b)=\{x \in X: a<x<b\}$ and $(\leftarrow, b)=\{x \in X: x<b\}$; analogously, $(a, \rightarrow)=\{x \in X: x>a\}$. The sets $(a, b)$ and $(\leftarrow, b)$ as well as $(a, \rightarrow)$ are called open intervals. If $X$ has the topology generated by its open intervals then it is called a linearly ordered topological space or LOTS. A subspace of a LOTS is called a generalized ordered space or simply GO space. A subset $I$ of a linearly ordered set $(X,<)$ is called convex if $(a, b) \subset I$ for any $a, b \in I$.

A family $\mathcal{A}$ is nested if either $A \subset A^{\prime}$ or $A^{\prime} \subset A$ for any $A, A^{\prime} \in \mathcal{A}$. A space $X$ is $l$-nested if it has a nested local base at every point. If $\mathcal{A}$ is a family of subsets of $X$ then $\triangle \mathcal{A}$ is the family of all finite intersections of the elements of $\mathcal{A}$. The family $\mathcal{A}$ is $T_{1}$-separating in $X$ if for any distinct $x, y \in X$ there is a set $A \in \mathcal{A}$ such that $x \in A \subset X \backslash\{y\}$.

The rest if our notation is standard and follows the book [13].

\section{DisCRETE REFLEXIVITY AND ORDER STRUCTURES}

Although local compactness, Čech-completeness and most local properties do not behave well even in countable spaces, we will show that they are still discretely reflexive in some nice classes.

Definition 3.1. Given a topological property $\mathcal{P}$, a space $X$ is called discretely $\mathcal{P}$ if $\bar{D}$ has $\mathcal{P}$ for any discrete set $D \subset X$.

Definition 3.2. A space $X$ is called discretely generated if for any set $A \subset X$, if $x \in \bar{A}$ then $x \in \bar{D}$ for some discrete $D \subset A$. The space $X$ is weakly discretely generated if for any non-closed set $A \subset X$, there exists a discrete $D \subset A$ such that $\bar{D} \backslash A \neq \emptyset$.

OBSERVATion 3.3. If $X$ is van Douwen's maximal space (see [10, Example 3.3]) then every discrete subspace of $X$ is closed so $X$ is a discretely locally compact (and hence discretely Čech-complete) space of first category. In particular, discrete Čech-completeness in countable spaces does not imply Cech-completeness or even the Baire property. Besides, $X$ is discretely metrizable without being a $k$-space. We will see that the situation both with local and global properties improves radically in generalized ordered spaces.

Proposition 3.4. If $X$ is weakly discretely generated then the k-property in $X$ is equivalent to discrete $k$-property.

Proof. Assume that $\bar{D}$ is a $k$-space for any discrete $D \subset X$. If $A$ is not closed in $X$ then there is a discrete $D \subset A$ such that $\bar{D} \backslash A \neq \emptyset$. The set $B=\bar{D} \cap A$ is not closed in $\bar{D}$ which is a $k$-space so we can find a compact set $K \subset \bar{D}$ such that $K \cap B$ is not closed in $K$; since $K \cap B=K \cap A$, the set $K$ witnesses the $k$-property of $X$. 
COROllary 3.5. If $X$ is a monotonically normal discretely $k$-space then $X$ is a $k$-space. In particular, every $G O$ space with discrete $k$-property is a $k$-space.

Proof. It was proved in [12, Theorem 2.10] that every monotonically normal space is discretely generated. Proposition 3.4 does the rest.

Proposition 3.6. If $X$ is weakly discretely generated and $t(\bar{D}) \leq \kappa$ for any discrete $D \subset X$ then $t(X) \leq \kappa$.

Proof. If $A$ is not closed in $X$ then there is a discrete $D \subset A$ such that $\bar{D} \backslash A \neq \emptyset$; choose a point $x \in \bar{D} \backslash A$. It follows from $t(\bar{D}) \leq \kappa$ that we can find a set $E \subset D$ such that $|E| \leq \kappa$ and $x \in \bar{E}$. Therefore $E \subset A$ and $\bar{E} \backslash A \neq \emptyset$, i.e. the set $E$ witnesses that $t(X) \leq \kappa$ (see [19, S.162, Lemma]).

Corollary 3.7. If $X$ is monotonically normal space and $t(\bar{D}) \leq \kappa$ for any discrete $D \subset X$ then $t(X) \leq \kappa$.

COROLlary 3.8. Tightness, character, pseudocharacter, sequentiality and the Fréchet-Urysohn property are discretely reflexive in GO spaces.

Proof. Every GO space is monotonically normal by [14, 5.21]; besides, in GO spaces character and pseudocharacter must coincide with tightness (see [2, Theorem 1.3.1]). Furthermore, sequentiality and Fréchet-Urysohn are both equivalent to countable tightness in GO spaces by the same [2, Theorem 1.3.1] so Corollary 3.7 shows that all our properties are discretely reflexive.

We will see that paracompactness is discretely reflexive in monotonically normal spaces. The following example shows that if we want it to be discretely reflexive in a class $\mathcal{P}$, then some restrictions must be imposed on $\mathcal{P}$.

EXAMPLE 3.9. There exists a space $X$ with the following properties:

(a) $X=M \cup E$ where $M$ is dense in $X$ and homeomorphic to $\mathbb{Q}$;

(b) the set $E$ is closed, discrete, disjoint from $M$ and $|E|=\mathfrak{c}$;

(c) if $D$ is discrete subset of $M$ then $\bar{D} \cap E=\emptyset$.

Therefore, $X$ is discretely paracompact non-normal space of countable $\pi$ weight.

Proof. Recall that $z \in \beta Z \backslash Z$ is a remote point of $Z$ if $z \notin \operatorname{cl}_{\beta Z}(D)$ for any nowhere dense set $D \subset Z$. Looking at the construction in the proof of Lemma 4.1 of van Douwen's paper [8], it is easy to see that

$(v D)$ if $\left\{U_{n}: n \in \omega\right\}$ is a discrete family of non-empty open subsets of $\mathbb{Q}$, then there exists a remote point $z \in \beta \mathbb{Q} \backslash \mathbb{Q}$ such that $z \in$ $\operatorname{cl}_{\beta \mathbb{Q}}\left(\bigcup_{n \in \omega} U_{n}\right)$ but $z \notin \operatorname{cl}_{\beta \mathbb{Q}}\left(\bigcup_{n \leq m} U_{n}\right)$ for any $m \in \omega$.

Fix an almost disjoint family $\mathcal{F}$ on the set $\omega$ such that $|\mathcal{F}|=\mathfrak{c}$. It is easy to find a disjoint family $\left\{U_{n}: n \in \omega\right\}$ of non-empty clopen subsets of $\mathbb{Q}$ such that $\mathbb{Q}=\bigcup_{n \in \omega} U_{n}$. For any $F \in \mathcal{F}$ let $G_{F}=\bigcup\left\{U_{n}: n \in F\right\}$ 
and apply the property $(v D)$ to find a remote point $p_{F} \in \beta \mathbb{Q} \backslash \mathbb{Q}$ such that $p_{F} \notin \operatorname{cl}_{\beta \mathbb{Q}}\left(\bigcup\left\{U_{n}: n \in A\right\}\right)$ for any finite $A \subset F$.

Consider the set $E=\left\{p_{F}: F \in \mathcal{F}\right\}$ and observe that $H_{F}=\operatorname{cl}_{\beta \mathbb{Q}}\left(G_{F}\right)$ is clopen in $\beta \mathbb{Q}$ for any $F \in \mathcal{F}$. It follows from our choice of $E$ that $H_{F} \cap E=$ $\left\{p_{F}\right\}$ for each $F \in \mathcal{F}$, i.e., $E$ is a discrete subset of $\beta \mathbb{Q}$. The set $P=$ $\operatorname{cl}_{\beta \mathbb{Q}}(E) \cap \mathbb{Q}$ is nowhere dense in $\mathbb{Q}$ so the set $M=\mathbb{Q} \backslash P$ is still homeomorphic to $\mathbb{Q}$.

It is clear that $E$ is a closed discrete subset of $X=M \cup E$ and $M \cap E=\emptyset$. Since all points of $E$ are remote, we also have the property $(c)$. Observing that $M$ is a dense second countable subset of $X$, we conclude that $\pi w(X)=\omega$. The statement after [13, Example 2.1.10] shows that the space $X$ is not normal.

To see that $X$ is discretely paracompact, take any discrete set $D \subset X$. Then $\bar{D}=Q \cup R$ where $Q=\overline{D \cap M}$ and $R=D \cap E$. It follows from the property $(c)$ that $Q \cap R=\emptyset$ and hence $\bar{D}$ is paracompact being the free union of a second countable space $Q$ and a discrete space $R$.

Proposition 3.10. If $X$ is a monotonically normal space and $\bar{D}$ is paracompact for any discrete $D \subset X$ then $X$ is paracompact. In other words, paracompactness is discretely reflexive in monotonically normal spaces.

Proof. Apply the result of Balogh and Rudin [5, Theorem I] which states that a monotonically normal space is paracompact if and only if no stationary subset of a regular uncountable cardinal is homeomorphic to a closed subset of $X$. Now, if $X$ is discretely paracompact and not paracompact then there is a closed subspace $S \subset X$ homeomorphic to a stationary subset of an uncountable regular cardinal. In particular, $S$ is scattered so we can find a discrete $D \subset S$ such that $S=\bar{D}$. Therefore $S$ is paracompact by discrete paracompactness of $X$; this is a contradiction with [5, Theorem I] applied to the embedding of $S$ in $S$.

It is an open problem of Arhangel'skii (see [3, Problem 14]) whether the Lindelöf property is discretely reflexive in any space. Arhangel'skii and Buzyakova established in [4] that discretely Lindelöf spaces of countable tightness are Lindelöf. It turns out that the Lindelöf property is discretely reflexive in monotonically normal spaces.

COROllary 3.11. If $X$ is a monotonically normal space and $\bar{D}$ is Lindelöf for any discrete $D \subset X$ then $X$ is Lindelöf. In other words, Lindelöfness is discretely reflexive in monotonically normal spaces.

Proof. If $X$ is a discretely Lindelöf monotonically normal space then it is paracompact by Proposition 3.10. Any discretely Lindelöf space trivially has countable extent and paracompact spaces of countable extent are Lindelöf so $X$ has to be Lindelöf. 
TheOREM 3.12. Suppose that a space $X$ has a nested base at a point $x$. Then $X$ is locally compact at $x$ if and only if $\bar{D}$ is locally compact at $x$ for any discrete $D \subset X$ with $x \in \bar{D}$.

Proof. Suppose that $X$ is not locally compact at a point $x$ but $\bar{D}$ is locally compact at $x$ for any discrete $D \subset X$ with $x \in \bar{D}$. For technical convenience, we also consider that $\bar{D}$ is locally compact at $x$ if $x \notin \bar{D}$. For some infinite regular cardinal $\kappa$ there exists a strictly decreasing local base $\left\{G_{\alpha}: \alpha<\kappa\right\}$ at the point $x$. Take a set $G \in \tau(x, X)$ such that $\bar{G} \subset G_{0}$ and observe that $\bar{G}$ is not compact; since compactness is discretely reflexive by [18, Lemma 4.13], there exists a discrete set $D \subset G$ such that $\bar{D}$ is not compact. It follows from local compactness of $\bar{D}$ at $x$ that we can find a set $U \in \tau(x, X)$ such that $\bar{U} \subset G$ and the closure of $D \cap \bar{U}$ is compact. As an immediate consequence, the set $D_{0}=D \backslash \bar{U}$ is discrete, $D_{0} \subset G_{0}$ and $\bar{D}_{0}$ is not compact; let $\mu_{0}=0$.

Proceeding inductively, assume that $\beta<\kappa$ and we have constructed discrete sets $\left\{D_{\alpha}: \alpha<\beta\right\}$ and ordinals $\left\{\mu_{\alpha}: \alpha<\beta\right\}$ with the following properties:

(1) $D_{\alpha} \subset G_{\mu_{\alpha}}$, and $x \notin \bar{D}_{\alpha}$ for each $\alpha<\beta$;

(2) if $\alpha<\gamma<\beta$ then $\mu_{\alpha}<\mu_{\gamma}$ and $D_{\alpha} \cap \bar{G}_{\mu_{\gamma}}=\emptyset$;

(3) the set $\bar{D}_{\alpha}$ is not compact for any $\alpha<\beta$.

Let $\mu=\sup \left\{\mu_{\alpha}: \alpha<\beta\right\}$; there exists an ordinal $\xi>\mu$ such that $\bar{G}_{\xi} \cap \bar{D}_{\alpha}=\emptyset$ for any $\alpha<\beta$. Take a set $H \in \tau(x, X)$ such that $\bar{H} \subset G_{\xi}$ and observe that $\bar{H}$ is not compact; since compactness is discretely reflexive by [18, Lemma 4.13], there exists a discrete set $E \subset H$ such that $\bar{E}$ is not compact. It follows from local compactness of $\bar{E}$ at the point $x$ that we can find a set $V \in \tau(x, X)$ such that $\bar{V} \subset H$ and the closure of $E \cap \bar{V}$ is compact. Let $D_{\beta}=E \backslash \bar{V}$ and $\mu_{\beta}=\xi$; it is immediate that the conditions (1)-(3) still hold for all $\alpha \leq \beta$ so our inductive procedure can be continued to construct a family $\mathcal{D}=\left\{D_{\alpha}: \alpha<\kappa\right\}$ and a $\kappa$-sequence $\left\{\mu_{\alpha}: \alpha<\kappa\right\}$ of ordinals such that the conditions (1) $-(3)$ are satisfied for all $\beta<\kappa$.

It follows from (2) that the family $\mathcal{W}=\left\{G_{\mu_{\alpha}} \backslash \bar{G}_{\mu_{\alpha+1}}: \alpha<\kappa\right\}$ is disjoint; since also $D_{\alpha} \subset G_{\mu_{\alpha}} \backslash \bar{G}_{\mu_{\alpha+1}}$ for all $\alpha<\kappa$, the set $D=\bigcup\left\{D_{\alpha}: \alpha<\kappa\right\}$ is discrete. Besides, the family $\mathcal{W}$ is a $\pi$-base at $x$ so every $U \in \tau(x, X)$ contains a set $D_{\alpha}$ for some $\alpha<\kappa$. Therefore every neighbourhood of $x$ in $\bar{D}$ contains some $D_{\alpha}$ which shows that no closure of a neighbourhood of $x$ in $\bar{D}$ is compact, i.e. $\bar{D}$ is not locally compact at $x$, a contradiction.

Corollary 3.13. If $X$ is an l-nested space and the closure of every discrete subspace of $X$ is locally compact then $X$ is locally compact.

Corollary 3.14. If $X$ is a first countable space and the closure of every discrete subspace of $X$ is locally compact then $X$ is locally compact. 
Corollary 3.15. If $X$ is a GO space and the closure of every discrete subspace of $X$ is locally compact then $X$ is locally compact.

Proof. There exists a linear order $<$ on $X$ for which there is a base in $X$ whose elements are convex. Fix a point $x \in X$ and observe that $x$ has a nested base in the space $R_{x}=\{y \in X: x \leq y\}$ so we can apply Theorem 3.12 to see that $x$ has a compact neighbourhood $V_{r}$ in $R_{x}$.

Analogously, $x$ has a nested base in the space $L_{x}=\{y \in X: y \leq x\}$ so we can apply Theorem 3.12 to see that $x$ has a compact neighbourhood $V_{l}$ in $L_{x}$. It is immediate that $V=V_{r} \cup V_{l}$ is a compact neighbourhood of $x$ in $X$ so $X$ is locally compact at $x$.

Theorem 3.16. If $X$ is a Borel set then $X$ is Čech-complete if and only if it is discretely Čech-complete.

Proof. Suppose that $X$ is a discretely Čech-complete non-Čech-complete Borel set and take a metrizable compactification $M$ of the space $X$. To see that $\mathbb{Q}$ is not discretely Cech-complete is a simple exercise and so, to obtain a contradiction, it suffices to find a closed copy of $\mathbb{Q}$ in $X$.

Since $X$ is not Cech-complete, the set $M \backslash X$ is not $\sigma$-compact; the set $M \backslash X$ is also Borel so we can apply [9, Lemma 8.8] to find a closed set $P$ of the space $M \backslash X$ homeomorphic to the irrationals. The set $G=\operatorname{cl}_{M}(P) \cap X$ is closed in $X$ and dense in $K=\operatorname{cl}_{M}(P)$. Besides, $P$ is Čech-complete so it is a $G_{\delta}$-subset of $K$ which shows that $G$ is $\sigma$-compact.

Every compact set $F \subset G$ misses $P$ which is dense in $K$ so it has empty interior; therefore it follows from $\sigma$-compactness of $G$ that $G$ is of first category in itself. This makes it possible to apply a classical theorem of Hurewicz (see [15]; an easier proof in English can be found in [11]) to conclude that $G$ (and hence $X$ ) has a closed subspace homeomorphic to $\mathbb{Q}$.

Corollary 3.17. A countable metric space is Čech-complete if and only if it is discretely Cech-complete.

Corollary 3.18. If a second countable space $X$ is discretely Čechcomplete then it has the Baire property.

Proof. Assume that $X$ is discretely Cech-complete and $P=\bigcap\left\{U_{n}: n \in\right.$ $\omega\}$ is not dense in $X$ for some family $\left\{U_{n}: n \in \omega\right\}$ of dense open subsets of $X$. The set $U=X \backslash \bar{P}$ is open in $X$, non-empty and has first category. Let $\mathcal{F}=\left\{F_{n}: n \in \omega\right\}$ be a family of closed nowhere dense subsets of $X$ such that $\bigcup \mathcal{F}=U$.

It is standard (see e.g. [21, Lemma 4.39]) to find a discrete set $D_{n} \subset U$ such that $F_{n} \subset \bar{D}_{n}$; as a consequence, every $F_{n}$ is a Borel set being closed in a Cech-complete space $\bar{D}_{n}$. Therefore $U$ is a Borel set being a countable union of Borel sets; besides an open subset of a discretely Čech-complete space must 
be discretely Čech-complete so $U$ is Čech-complete by Theorem 3.16. Thus, $U$ is a Cech-complete space of first category which is a contradiction.

Corollary 3.19. A metrizable space is scattered if and only if it is discretely scattered.

Proof. Suppose that $X$ is a metrizable discretely scattered space. If $X$ is not scattered then it is standard to find a crowded countable set $Y \subset X$. It was proved in [17] that a countable second countable space is Cech-complete if and only if it is scattered so the closure in $Y$ of every discrete $D \subset Y$ is Cech-complete. Applying Corollary 3.17 we conclude that $Y$ is Čech-complete and hence scattered, which is a contradiction.

THEOREM 3.20. If a GO space is discretely scattered, then it is scattered.

Proof. Suppose that $X$ is a discretely scattered subspace of a LOTS; then we can fix a linear order $<$ on $X$ such that $X$ has a base consisting of convex sets. Recall that every GO space is hereditarily collectionwise normal so

(*) if $D$ is a discrete subset of $X$ then we can find an open convex set $U_{d} \ni d$ for any $d \in D$ such that the family $\left\{\bar{U}_{d}: d \in D\right\}$ is disjoint.

We will also need the following property.

(**) If $\mathcal{A}$ is a family of disjoint convex open sets such that $x \notin \bar{A}$ for any $A \in \mathcal{A}$ and $x \in \overline{\cup \mathcal{A}}$ then $\mathcal{A}$ is a $\pi$-base at $x$.

To see that (**) holds, let $L=\{y \in X: y<x\}$ and $R=\{y \in X: y>x\}$ and observe that either $A \subset L$ or $A \subset R$ for any $A \in \mathcal{A}$ so we can assume, without loss of generality, that $A \subset R$ for all $A \in \mathcal{A}$. Take any convex $V \in \tau(x, X)$; there exists $A \in \mathcal{A}$ with $A \cap V \neq \emptyset$. Pick a point $a \in A \cap V$. The set $(\leftarrow, a)$ being open in $X$, we can find $B \in \mathcal{A} \backslash\{A\}$ for which $B \cap(x, a) \neq \emptyset$; it easily follows from the fact that $\mathcal{A}$ is disjoint and convexity of $B$ that $B \subset(x, a) \subset V$ so $(* *)$ is proved.

Now assume that $X$ is not scattered and fix a crowded set $Y \subset X$. Call a set $A \subset X$ adequate if $A$ is open, convex and $A \cap Y \neq \emptyset$. It is clear that every adequate set has an infinite intersection with $Y$. We will inductively construct a non-empty discrete set $D_{n} \subset Y$ and a disjoint family $\mathcal{E}_{n}$ of adequate sets for each $n \in \omega$ in such a way that

(4) $D_{n} \subset \bar{D}_{n+1} \backslash D_{n+1}$ for any $n \in \omega$;

(5) $D_{n} \subset \overline{\cup \mathcal{E}_{n}} \backslash \bigcup\left\{\bar{E}: E \in \mathcal{E}_{n}\right\}$ for any $n \in \omega$;

(6) if $n \in \omega$ then $D_{i} \cap\left(\bigcup\left\{\bar{E}: E \in \mathcal{E}_{j}\right\}\right)=\emptyset$ whenever $i, j \leq n$;

(7) the family $\bigcup_{i \leq n} \mathcal{E}_{i}$ is disjoint for any $n \in \omega$;

(8) there exists a set $G_{n} \in \tau(X)$ such that $G_{n} \cap\left(\left(\bigcup_{i \leq n} \mathcal{E}_{i}\right) \cup D_{n}\right)=\emptyset$ and $D_{n} \subset \overline{G_{n} \cap Y} \backslash G_{n}$ for every $n \in \omega$. 
If we succeed in constructing the promised sets and families, then it follows from (4) that $Z=\bigcup_{n \in \omega} D_{n}$ is a crowded subset of $X$. If $\mathcal{E}=\bigcup_{n \in \omega} \mathcal{E}_{n}$ then the family $\mathcal{E}$ is disjoint by (7) and the properties (4)-(6) show that $(\bigcup\{\bar{E}: E \in \mathcal{E}\}) \cap Z=\emptyset$ and $Z \subset \overline{\bigcup \mathcal{E}}$. This, together with the property $(* *)$ implies that if we choose a point $x_{E} \in E$ for any $E \in \mathcal{E}$ then $D=\left\{x_{E}: E \in \mathcal{E}\right\}$ is a discrete set such that $Z \subset \bar{D}$ which is a contradiction with the fact that $\bar{D}$ is scattered.

To start off, take any point $x_{0} \in Y$ and let $D_{0}=\left\{x_{0}\right\}$. Since $X$ is discretely generated by [12, Theorem 2.10], we can find a discrete set $P \subset Y$ such that $x_{0} \in \bar{P} \backslash P$. Apply hereditary collectionwise normality of $X$ to find an adequate set $U_{p}$ such that $p \in U_{p}$ and $x_{0} \notin \bar{U}_{p}$ for every $p \in P$ while the family $\left\{\bar{U}_{p}: p \in P\right\}$ is disjoint. It is easy to find disjoint adequate sets $V_{p}, W_{p}$ such that $V_{p} \cup W_{p} \subset U_{p}$ for each $p \in P$. Now, letting $\mathcal{E}_{0}=\left\{V_{p}: p \in P\right\}$ and $G_{0}=\bigcup\left\{W_{p}: p \in P\right\}$ we can use (**) to see that the sets $D_{0}, G_{0}$ and the family $\mathcal{E}_{0}$ have the properties (5)-(8) and the property (4) holds vacuously.

Suppose that $m \in \omega$ and we have sets $D_{0}, G_{0}, \ldots, D_{m}, G_{m}$ and the families $\mathcal{E}_{0}, \ldots, \mathcal{E}_{m}$ with the properties (4)-(8). Apply hereditary collectionwise normality of $X$ to find an adequate set $U_{x}$ such that $x \in U_{x}$ for every $x \in D_{m}$ and the family $\left\{\bar{U}_{x}: x \in D_{m}\right\}$ is disjoint. Since $x \in \overline{U_{x} \cap G_{m} \cap Y}$, we can find a discrete set $P_{x} \subset U_{x} \cap G_{m} \cap Y$ such that $x \in \bar{P}_{x}$. For every $y \in P_{x}$ pick an adequate set $A(y, x)$ such that $y \in A(y, x) \subset \overline{A(y, x)} \subset U_{x} \cap G_{m}$ and the family $\left\{\overline{A(y, x)}: y \in P_{x}\right\}$ is disjoint.

It is easy to find disjoint adequate sets $W(y, x)$ and $V(y, x)$ such that $W(y, x) \cup V(y, x) \subset A(y, x)$; choose a point $b(y, x) \in V(y, x)$ for any $y \in P_{x}$. The set $D_{m+1}=\left\{b(y, x): x \in D_{m}\right.$ and $\left.y \in P_{x}\right\}$ is easily seen to be discrete and it follows from $(* *)$ that $x \in \overline{\left\{b(y, x): y \in P_{x}\right\}}$ for any $x \in D_{m}$ whence $D_{m} \subset \bar{D}_{m+1}$. Letting $\mathcal{E}_{m+1}=\left\{W(y, x): x \in D_{m}\right.$ and $\left.y \in P_{x}\right\}$ and applying $(* *)$ again we can see that (5) holds for $n=m+1$. It follows from $\bigcup\{\bar{E}: E \in$ $\left.\mathcal{E}_{m+1}\right\} \subset G_{m}$ that the conditions (6) and (7) are also satisfied for $n=m+1$.

Using once more the fact that $V(y, x) \cap Y$ is infinite, we can find an adequate set $H(y, x) \subset \overline{H(y, x)} \subset V(y, x)$ such that $b(y, x) \notin \overline{H(y, x)}$ for any $x \in D_{m}$ and $y \in P_{x}$; let $G_{m+1}=\bigcup\left\{H(y, x): x \in D_{m}, y \in P_{x}\right\}$. It is straightforward that the conditions (4)-(8) are now satisfied for all $n \leq m+1$ and hence our inductive procedure can be continued to construct the promised sets and families.

Recall that an uncountable space $X$ is a Luzin space if it is dense in itself and every nowhere dense subset of $X$ is countable. The following example was originally constructed under $\mathrm{CH}$. The authors are grateful to the referee who indicated that the same idea works for any Luzin space. Since the existence of a Luzin space is a weaker assumption than $\mathrm{CH}$, we have a more general result with an easier proof. 
ExAMPLE 3.21. If there exists a Luzin set $L \subset \mathbb{R}$, then there exists a set $X \subset \mathbb{R}$ which is discretely Čech-complete but not Čech-complete.

Proof. Our space will be $X=\bar{L} \backslash L$; it is easy to see that $X$ is dense in $\bar{L}$. The space $X$ is not Čech-complete for otherwise $X$ is a $G_{\delta}$-subset of $\bar{L}$ and hence $L$ is an $F_{\sigma}$-subset of $\mathbb{R}$ so $L$ contains an uncountable closed subset of $\mathbb{R}$ which is impossible.

If $N$ is a closed nowhere dense subspace of $X$ then $P=\bar{N}$ is closed nowhere dense in $\bar{L}$ so $P \cap L$ is nowhere dense in $L$ and hence countable. As a consequence, $N$ is a $G_{\delta}$-subset of a Čech-complete space $P$. Therefore every closed nowhere dense subset of $X$ is Cech-complete. Since $\bar{D}$ is nowhere dense in $X$ for any discrete $D \subset X$, we conclude that $X$ is discretely Cech-complete.

LEMMA 3.22. If $X$ is a LOTS and $\mathcal{U}$ a $T_{1}$-separating family of open intervals of $X$ then $\bigwedge \mathcal{U}$ is a base of $X$.

Proof. Take any $x, a, b \in X$ with $x \in(a, b)$. We can find $U, V \in \mathcal{U}$ such that $x \in U \cap V$ while $b \notin U$ and $a \notin V$. If $y \in U \cap V$ and $y \geq b$ then it follows from $\{x, y\} \subset U$ and $b \in(x, y]$ that $b \in U$ because $U$ is a convex set. This contradiction shows that $y<b$. Analogously, $y \leq a$ implies that $a \in V$ which is a contradiction. Therefore $a<y<b$ for any point $y \in U \cap V \in \wedge \mathcal{U}$, i.e., $x \in U \cap V \subset(a, b)$. The cases $x \in(\leftarrow, b)$ and $x \in(a, \rightarrow)$ are easier.

LEMma 3.23. Suppose that a linearly ordered space $X$ is either finite or we have the equality $w(I)=w(X)$ for any non-empty open interval $I \subset X$. Then there exists a discrete set $D \subset X \times X$ such that $\Delta_{X} \subset \bar{D}$.

Proof. If $X$ is finite then it is discrete so $\Delta=\Delta_{X}$ is discrete as well and hence there is nothing to prove. If $X$ is infinite then the cardinal $\kappa=w(X)$ is also infinite. Fix a base $\mathcal{B}$ in $X$ such that every $U \in \mathcal{B}$ is a non-empty open interval and $|\mathcal{B}|=\kappa$; let $\left\{G_{\alpha}: \alpha<\kappa\right\}$ be an enumeration of $\mathcal{B}$.

Take a point $x_{0} \in G_{0}$; pick any $y_{0} \in G_{0} \backslash\left\{x_{0}\right\}$ and let $z_{0}=\left(x_{0}, y_{0}\right)$. We can find disjoint open intervals $U_{0}$ and $V_{0}$ such that $x_{0} \in U_{0}$ and $y_{0} \in V_{0}$. Proceeding by induction let $z_{0}=\left(x_{0}, y_{0}\right)$ and assume that $\alpha<\kappa$ and we have a set $\left\{z_{\beta}: \beta<\alpha\right\}$ and a family $\left\{U_{\beta}, V_{\beta}: \beta<\alpha\right\}$ of open intervals with the following properties:

(9) $z_{\beta}=\left(x_{\beta}, y_{\beta}\right) \in(X \times X) \backslash \Delta$ for any $\beta<\alpha$;

(10) $x_{\beta} \in U_{\beta}, y_{\beta} \in V_{\beta}$ and $U_{\beta} \cap V_{\beta}=\emptyset$ for every $\beta<\alpha$;

(11) $z_{\beta} \notin \overline{\left\{z_{\gamma}: \gamma<\beta\right\}}$ for all $\beta<\alpha$;

(12) $z_{\beta} \notin H_{\beta}=\bigcup\left\{U_{\gamma} \times V_{\gamma}: \gamma<\beta\right\}$ for each $\beta<\alpha$;

(13) $\left\{z_{\gamma}: \gamma \leq \beta\right\} \cap\left(G_{\beta} \times G_{\beta}\right) \neq \emptyset$ for any $\beta<\alpha$.

Let $D_{\alpha}=\left\{z_{\beta}: \beta<\alpha\right\}$; if $D_{\alpha} \cap(B \times B) \neq \emptyset$ for any $B \in \mathcal{B}$ then we set $D=D_{\alpha}$ and stop the induction. If $D_{\alpha}$ does not meet $B \times B$ for some $B \in \mathcal{B}$, then the property (13) shows that $\gamma=\min \left\{\beta: D_{\alpha} \cap\left(G_{\beta} \times G_{\beta}\right)=\emptyset\right\} \geq \alpha$. 
Suppose that the set $\left(G_{\gamma} \times G_{\gamma}\right) \backslash \Delta$ is contained in $H_{\alpha}=\bigcup\left\{U_{\beta} \times V_{\beta}: \beta<\right.$ $\alpha\}$. Then the family $\mathcal{H}=\left\{U_{\beta} \cap G_{\gamma}, V_{\beta} \cap G_{\gamma}: \beta<\alpha\right\}$ is $T_{1}$-separating in $G_{\gamma}$. If $\kappa=\omega$ then we obtain a finite $T_{1}$-separating family on an infinite set; it is easy to see that this is impossible. If $\kappa>\omega$ then it follows from Lemma 3.22 that $w\left(G_{\gamma}\right) \leq|\mathcal{H}| \cdot \omega<\kappa$ which is a contradiction.

Therefore $\left(G_{\gamma} \times G_{\gamma}\right) \backslash \Delta$ is not contained in $H_{\alpha}$ and hence we can find distinct points $x_{\alpha}, y_{\alpha} \in G_{\gamma}$ such that $z_{\alpha}=\left(x_{\alpha}, y_{\alpha}\right) \notin H_{\alpha}$. Choose disjoint open intervals $U_{\alpha}, V_{\alpha}$ such that $x_{\alpha} \in U_{\alpha}$ and $y_{\alpha} \in V_{\alpha}$. It is immediate that the conditions (9)-(13) are still satisfied for all $\beta \leq \alpha$.

For some $\alpha \leq \kappa$ our inductive procedure will conclude and we will have the set $D=D_{\alpha}$; by our choice of $D$ (and the property (13) if $\alpha=\kappa$ ) we have $D \cap(B \times B) \neq \emptyset$ for any $B \in \mathcal{B}$. This trivially implies that $\Delta \subset \bar{D}$ so it suffices to show that $D_{\alpha}$ is discrete for any $\alpha \leq \kappa$. Fix any $\beta<\alpha$ and observe that it follows from (11) that $z_{\beta} \notin \overline{\left\{z_{\gamma}: \gamma<\beta\right\}}$. Besides, we have the inclusion $\left\{z_{\gamma}: \gamma>\beta\right\} \subset(X \times X) \backslash\left(U_{\beta} \times V_{\beta}\right)$ by the property (12) so $z_{\beta} \notin \overline{\left\{z_{\gamma}: \gamma>\beta\right\}}$ and hence the set $D_{\alpha}$ is discrete.

OBSERVATION 3.24. In [6] it was established that for an arbitrary Lindelöf $p$-space $X$ it is possible to find a discrete subset $D \subset X \times X$ such that $\Delta_{X}=\{(x, x): x \in X\} \subset \bar{D}$. In particular, if $X$ is a Lindelöf $p$-space and $X \times X$ is discretely Čech-complete then $X$ is Čech-complete. It was also proved in [6] that under $\mathrm{CH}$ there exists a crowded countable space $X$ such that every discrete set $D \subset X \times X$ is closed. In particular, $X \times X$ is discretely Čech-complete space of first category.

EXAmPle 3.25. It was proved in [20, Theorem 6.14] that under the negation of the Souslin Hypothesis there exists a Souslin line $L$ with a pointcountable base. It is easy to see that $\bar{A}$ is second countable for any countable $A \subset L$. Furthermore, $L$ is hereditarily Lindelöf so every discrete $D \subset L$ is countable and therefore $\bar{D}$ is second countable. Therefore $L$ is a discretely second countable LOTS which fails to be separable.

THEOREM 3.26. If $X$ is a LOTS then there exists a discrete set $D \subset X \times X$ such that $\Delta_{X} \subset \bar{D}$.

Proof. Call a non-empty open interval $I \subset X$ adequate if $w(J)=w(I)$ for any non-empty open interval $J \subset I$. If $U$ is a non-empty open subset of $X$ then we can choose a non-empty open interval $I \subset U$ of minimal weight; it is immediate that $I$ is adequate so adequate intervals form a $\pi$-base of $X$.

Take a maximal disjoint family $\mathcal{I}$ of adequate intervals; then $\bigcup \mathcal{I}$ is dense in $X$. Observe that Lemma 3.23 is applicable to any adequate interval so there exists a discrete set $D_{I} \subset I \times I$ such that $\Delta_{I} \subset \bar{D}_{I}$ for every $I \in \mathcal{I}$. It is straightforward that $D=\bigcup\left\{D_{I}: I \in \mathcal{I}\right\}$ will be a discrete subset of $X \times X$ such that $\Delta_{X} \subset \bar{D}$. 
COROLlary 3.27. If $\mathcal{P}$ is a topological property which is closed-hereditary and $X$ is a LOTS such that $X \times X$ is discretely $\mathcal{P}$ then $X$ has $\mathcal{P}$. In particular, if $X \times X$ is discretely Čech-complete (discretely metrizable or discretely zero-dimensional) then $X$ is Čech-complete (metrizable or zerodimensional respectively).

Proof. Apply Theorem 3.26 to find a discrete subspace $D \subset X \times X$ such that $\Delta_{X} \subset \bar{D}$. The set $\Delta_{X}$ must have $\mathcal{P}$ because it is closed in the space $\bar{D}$ so $X$ has $\mathcal{P}$ being homeomorphic to $\Delta_{X}$.

\section{OPEN PROBLEMS}

Discrete reflexivity of topological properties turned out to be an interesting topic with a potential to provide new information about local compactness and Čech-completeness even in second countable spaces. That there are still a lot of relevant facts to be discovered can be seen from the following list of open questions.

QUESTION 4.1. Suppose that $X$ is an analytic second countable discretely Čech-complete space. Must it be Čech-complete?

Question 4.2. Does there exist in ZFC a second countable discretely Čech-complete space which is not Čech-complete?

Question 4.3. Does there exist in ZFC a metrizable discretely Čechcomplete space which is not $\check{C}$ ech-complete?

QUESTION 4.4. Suppose that $X$ is a monotonically normal discretely locally compact space. Must $X$ be locally compact?

QUESTION 4.5. Suppose that $X$ is a monotonically normal discretely locally compact space. Must $X$ be Čech-complete?

Question 4.6. Suppose that $X$ is a monotonically normal discretely locally compact space. Must $X$ have the Baire property?

Question 4.7. Suppose that $X$ is a monotonically normal discretely scattered space. Must $X$ be scattered?

Question 4.8. Suppose that $X$ is a monotonically normal discretely Cech-complete space. Must $X$ have the Baire property?

Question 4.9. Suppose that $X$ is a linearly ordered discretely Čechcomplete space. Must $X$ have the Baire property?

QUESTION 4.10. Suppose that $X$ is a GO space. Is it true that there exists a discrete set $D \subset X \times X$ such that $\Delta_{X} \subset \bar{D}$ ?

Question 4.11. Suppose that $X$ is a monotonically normal space. Is it true that $X^{\omega}$ is d-separable? 
QUeSTION 4.12. Suppose that $X$ is a monotonically normal space and $X \times X$ is discretely Cech-complete. Must $X$ be Čech-complete?

QUESTION 4.13. Suppose that $X$ is a monotonically normal space and $X \times X$ is discretely locally compact. Must $X$ be locally compact?

QUESTION 4.14. Suppose that $X$ is a monotonically normal space and $X \times X$ is discretely zero-dimensional. Must $X$ be zero-dimensional?

Question 4.15. Suppose that $X$ is a monotonically normal space and $X \times X$ is discretely scattered. Must $X$ be scattered?

\section{ACKNowledgements.}

Research supported by Promep 912011, Proyecto 12611768 (Mexico) and by CONACyT grant CB-2012-01-178103 (Mexico)

\section{REFERENCES}

[1] O. Alas, V.V. Tkachuk, R.G. Wilson, Closures of discrete sets often reflect global properties, Topology Proc. 25 (2000), 27-44.

[2] A. V. Arhangel'skii, Structure and classification of topological spaces and cardinal invariants, (in Russian), Uspehi Mat. Nauk 33 (1978), 29-84.

[3] A. V. Arhangel'skiř, A generic theorem in the theory of cardinal invariants of topological spaces, Comment. Math. Univ. Carolin. 36 (1995), 303-325.

[4] A. V. Arhangel'skii and R. Z. Buzyakova, On linearly Lindelöf and strongly discretely Lindelöf spaces, Proc. Amer. Math. Soc. 127 (1999), 2449-2458.

[5] Z. Balogh and M. E. Rudin, Monotone normality, Topology Appl. 47 (1992), 115-127.

[6] D. Burke and V. V. Tkachuk, Diagonals and discrete subsets of squares, Comment. Math. Univ. Carolin. 54 (2013), 69-82.

[7] D. Burke and V. V. Tkachuk, Discrete reflexivity and complements of the diagonal, Acta Math. Hungar. 139 (2013), 120-133.

[8] E. K. van Douwen, Remote points, Dissertationes Math. (Rozprawy Mat.) 188 (1981), $45 \mathrm{pp}$.

[9] E. K. van Douwen, The integers and topology, in: Handbook of Set-Theoretic Topology, ed. by K. Kunen and J.E. Vaughan, Elsevier S.P., Amsterdam, 1984, 111-167.

[10] E. K. van Douwen, Applications of maximal topologies, Topology Appl. 51 (1993), $125-139$.

[11] E. K. van Douwen, Closed copies of the rationals, Comment. Math. Univ. Carolin. 28 (1987), 137-139.

[12] A. Dow, M. G. Tkachenko, V. V. Tkachuk and R. G. Wilson, Topologies generated by discrete subspaces, Glas. Mat. Ser. III 37(57) (2002), 189-212.

[13] R. Engelking, General topology, PWN, Warszawa, 1977.

[14] G. Gruenhage, Generalized metric spaces, in: Handbook of set-theoretic topology, North Holland, New York, 1984, 423-501.

[15] W. Hurewicz, Relativ perfekte Teile von Punktmengen und Mengen (A), Fund. Math. 12 (1928), 78-109.

[16] I. Juhász and Z. Szentmiklossy, On d-separability of powers and $C_{p}(X)$, Topology Appl. 155 (2008), 277-281.

[17] B. Knaster and K. Urbanik, Sur les espaces complets séparables de dimension 0, Fund. Math. 40 (1953), 194-202. 
[18] V. V. Tkachuk, Spaces that are projective with respect to classes of mappings, Trans. Moscow Math. Soc. 50 (1988), 139-156.

[19] V. V. Tkachuk, A $C_{p}$-theory problem book. Topological and function spaces, Springer, New York, 2011.

[20] S. Todorčević, Trees and linearly ordered sets, in: Handbook of set-theoretic topology, North Holland, New York, 1984, 235-293.

[21] R. C. Walker, The Stone-Čech compatification, Springer-Verlag, New York, 1974.

V. V. Tkachuk

Departamento de Matemáticas

Universidad Autónoma Metropolitana

Av. San Rafael Atlixco, 186, Col. Vicentina, Iztapalapa

C.P. 09340, Mexico D.F.

Mexico

E-mail: vova@xanum.uam.mx

R. G. Wilson

Departamento de Matemáticas

Universidad Autónoma Metropolitana

Av. San Rafael Atlixco, 186, Col. Vicentina, Iztapalapa

C.P. 09340, Mexico D.F.

Mexico

E-mail: rgw@xanum.uam.mx

Received: 2.8.2013.

Revised: 8.12.2013. 